CLINICAL STUDY

\title{
Thyroid hormone status within the physiological range affects bone mass and density in healthy men at the age of peak bone mass
}

\author{
Greet Roef $^{1,2}$, Bruno Lapauw ${ }^{1,2}$, Stefan Goemaere $^{2}$, Hans Zmierczak ${ }^{2}$, Tom Fiers ${ }^{3}$, Jean-Marc Kaufman ${ }^{1,2,3}$ \\ and Youri Taes ${ }^{1,2}$ \\ ${ }^{1}$ Department of Endocrinology, ${ }^{2}$ Unit for Osteoporosis and Metabolic bone diseases and ${ }^{3}$ Laboratory of Hormonology, Department of Clinical Chemistry, \\ Ghent University Hospital, De Pintelaan 185, 9000 Ghent, Belgium
}

(Correspondence should be addressed to G Roef; Email: greet.roef@ugent.be)

\begin{abstract}
Context: The hormonal factors involved in the regulation of peak bone mass (PBM) in men have not been fully investigated. Apart from gonadal steroids and somatotropic hormones, thyroid hormones are known to affect bone maturation and homeostasis and are additional candidate determinants of adult bone mass.

Objective: We aimed to investigate between-subject physiological variation in free and total thyroid hormone concentrations, TSH, and thyroid binding globulin (TBG) in relation to parameters of bone mass, geometry, and mineral density in healthy men at the age of PBM.

Design and setting: We recruited 677 healthy male siblings aged 25-45 years in a cross-sectional, population-based study. Areal and volumetric bone parameters were determined using dual-energy x-ray absorptiometry (DXA) and peripheral quantitative computed tomography (pQCT). Total and free thyroid hormones, TBG, and TSH were determined using immunoassays.

Results: Free and total thyroid hormone concentrations were inversely associated with bone mineral density (BMD) and bone mineral content $(\mathrm{BMC})$ at the hip and total body (free triiodothyronine $\left(\mathrm{FT}_{3}\right)$, total $\mathrm{T}_{3}\left(\mathrm{TT}_{3}\right)$, and total $\mathrm{T}_{4}\left(\mathrm{TT}_{4}\right)$ ) and at the spine $\left(\mathrm{FT}_{3}\right)$. TBG was negatively associated with BMC and areal BMD at all sites. At the radius, cortical bone area was inversely associated with $\mathrm{TT}_{3}, \mathrm{TT}_{4}$, and TBG, and trabecular bone density was inversely associated with free thyroxine, $\mathrm{TT}_{4}$, and TBG. We observed inverse associations between cortical bone area at the mid-tibia and $\mathrm{FT}_{3}, \mathrm{TT}_{3}, \mathrm{TT}_{4}$, and TBG. No associations between TSH and DXA or pQCT measurements were found.

Conclusion: In healthy men at the age of PBM, between-subject variation in thyroid hormone concentrations affects bone density, with higher levels of $\mathrm{FT}_{3}, \mathrm{TT}_{3}, \mathrm{TT}_{4}$, and $\mathrm{TBG}$ being associated with less favorable bone density and content.
\end{abstract}

European Journal of Endocrinology 164 1027-1034

\section{Introduction}

Peak bone mass (PBM) in young adults is a major determinant of bone mass later in life (1). Besides environmental factors such as exercise, smoking, and nutrition, genetic factors play a major role. These genetic and environmental influences are mediated in part by hormonal regulation of bone accrual during growth and maturation (1-3). The major and most extensively studied hormonal systems implicated in this regulation are the somatotropic and the gonadal axes $(3,4)$. Another candidate hormonal determinant of PBM is thyroid hormone, known to have potentially marked effects on bone maturation and metabolism.

Thyroid hormone is essential for normal growth and bone development. Thyroid hormone deficiency results in delayed skeletal development, delayed bone age, and growth arrest accompanied by epiphyseal dysgenesis (5). Hyperthyroidism in childhood, in contrast, induces not only accelerated skeletal development and growth with advanced bone age but also early premature fusion of the epiphyseal growth plates and cessation of growth (6).

In adults with hypothyroidism, bone turnover is reduced with a prolonged bone formation phase that leads to an increased mineralization phase and an apparent increase of bone mineral density (BMD). Hyperthyroidism in adulthood, on the other hand, is associated with increased bone turnover and a reduction in BMD at various skeletal sites due to increased cortical porosity and accelerated bone loss (7-11). Interestingly, population studies have shown that both hypo- and hyper-thyroidism in adults may be associated with an increased fracture risk (12). 
However, little is known about the influence of substantial variation in thyroid parameters within the physiological euthyroid range on bone density and geometry. The effects of variation across the normal range of thyroid status on areal BMD (aBMD) and fracture susceptibility in middle-aged and older subjects were described in two recent studies. The first study reported the effects in postmenopausal women (13) and the second study in men and women above the age of 55 years (14). To our present knowledge, no studies have addressed the issue of influence of variation across the normal range of thyroid hormone status on PBM in male. We therefore studied the relationship between indices of thyroid hormone status within the physiological range and aBMD, volumetric BMD (vBMD) and bone geometry in healthy young male siblings at the age of PBM.

\section{Materials and methods}

\section{Study design and population}

A total of 767 young men were recruited from three semi-rural to suburban communities around Ghent, Belgium. Men aged 25-45 years were contacted by mail and asked whether they had a brother in the same age range also willing to participate. The study design and population characteristics have been previously described $(3,4,15)$. The general aim of the study was to investigate the genetic and environmental determinants of PBM in men and therefore we sampled brothers together with their parents. For this publication, we only considered the brothers. After exclusions, 296 pairs of brothers were included in the study; 64 men were included as single participants, as their brothers could not participate in the study, 19 men were included as third brother in a family, and two as fourth brother. All brother analyses were done by family structure. The maximal age difference between brother pairs was arbitrarily set at 12 years. All participants were in good health and completed questionnaires about previous illness, lifestyle, physical activity, education, profession, nutrition, and smoking. Exclusion criteria were defined as illnesses or use of medication affecting body composition, hormone or bone metabolism: current or prolonged ( $>3$ months) use of glucocorticosteroids, anti-androgens, vitamin D supplements, insulin, thyroxine $\left(\mathrm{T}_{4}\right)$, and previous or current use of anti-epileptic drugs, hypogonadism, hyper- or hypothyroidism, cystic fibrosis, malabsorption or eating disorders, disorders of collagen metabolism or bone development, chronic renal failure, alcohol abuse, and autoimmune rheumatoid disease. The study protocol was approved by the ethical committee of the Ghent University Hospital, and informed consent was obtained from all participants. Smoking habits were registered as current or previous smoking.

\section{Anthropometry and aBMD}

Standing height was measured using a wall-mounted Harpenden stadiometer (Holtain Ltd, Crymych, UK). Body weight was measured in light indoor clothing without shoes. Anthropometric measurements were performed as described previously (4) according to the Anthropometric Standardization Reference Manual (16). aBMD at the lumbar spine and proximal femur (total hip region) of the nondominant limb was measured using dual-energy x-ray absorptiometry (DXA) with a Hologic QDR-4500A device (software version 11.2.1; Hologic, Bedford, MA, USA). The coefficient of variation $(\mathrm{CV})$ was $<1 \%$ as calculated from daily spine phantom measurements.

\section{vBMD and bone geometry}

A peripheral quantitative computed tomography (pQCT) device (XCT-2000, Stratec Medizintechnik, Pforzheim, Germany) was used to scan the dominant leg (tibia) and forearm (radius). The dominant side was selected to allow the assessment of the relationship between muscle area and bone parameters. The cortical volumetric BMD (vBMD; $\mathrm{mg} / \mathrm{cm}^{3}$ ), cortical cross-sectional area $\left(\mathrm{mm}^{2}\right)$, endosteal and periosteal circumferences, and cortical thickness $(\mathrm{mm})$ were measured at the mid-radius $(66 \%$ of bone length from the distal end) and mid-tibia (66\%). Trabecular vBMD $\left(\mathrm{mg} / \mathrm{cm}^{3}\right)$ was measured using a scan through the metaphysis (at $4 \%$ of bone length) at the nondominant radius. The $\mathrm{CV}$ for the calibration phantom was $<1 \%$ as calculated form daily phantom measurements.

\section{Biochemical determinations}

Venous blood samples were obtained between 0800 and $1000 \mathrm{~h}$ after overnight fasting. All serum samples were stored at $-80{ }^{\circ} \mathrm{C}$ until batch analysis for parameters of thyroid function and bone metabolism.

Thyroid parameters included $\mathrm{TSH}$, free $\mathrm{T}_{4}\left(\mathrm{FT}_{4}\right)$, free triiodothyronine $\left(\mathrm{FT}_{3}\right)$, total $\mathrm{T}_{3}\left(\mathrm{TT}_{3}\right)$, total $\mathrm{T}_{4}$ $\left(\mathrm{TT}_{4}\right)$, thyroid binding globulin (TBG), as well as thyroperoxidase antibodies (TPOAb) and thyroglobulin antibodies (TgAb). Parameters of bone metabolism consisted of C-terminal telopeptide of type I collagen (CTX) and procollagen type 1 amino-terminal propeptide (P1NP). Commercial RIAs were used to determine serum levels of total T, sex hormone binding globulin (SHBG; Orion Diagnostica, Espoo, Finland), and estradiol ( $\mathrm{E}_{2}$; Clinical Assay; DiaSorin, Saluggia, Italy).

All thyroid parameter measurements with the exception of $\mathrm{TT}_{3}, \mathrm{TT}_{4}$, and TBG were performed using an immuno-electrochemiluminescence technique (Modular E 170, Roche Diagnostics GmbH). This assay has proven to give comparable results with other assays, according to the report from the IFCC WG for standardization of thyroid function tests (17). 
Table 1 General characteristics and thyroid parameters of study participants $(n=677)$ before exclusion of persons with positive thyroid autoimmunity. Variables are given as mean \pm s.D. and when there was a non-Gaussian distribution, data were presented as median (first to third quartile).

\begin{tabular}{lll}
\hline Parameter & Values & $\begin{array}{l}\text { Reference } \\
\text { range }\end{array}$ \\
\hline Age (years) & $34.3 \pm 5.5$ & \\
Height $(\mathrm{cm})$ & $179 \pm 7$ & \\
Weight $(\mathrm{kg})$ & $81 \pm 12$ & \\
$\mathrm{BMl}\left(\mathrm{kg} / \mathrm{m}^{2}\right)$ & $25.2 \pm 3.6$ & \\
$\mathrm{Smokers}(\%)$ & 22.9 & $0.27-4.2$ \\
TSH $(\mu \mathrm{U} / \mathrm{ml})$ & $1.5(1.14-2.14)$ & $250-440$ \\
Free $\mathrm{T}_{3}(\mathrm{pg} / \mathrm{dl})$ & $391 \pm 37$ & $0.9-1.7$ \\
Free $\mathrm{T}_{4}(\mathrm{ng} / \mathrm{dl})$ & $1.44 \pm 0.18$ & $87-180$ \\
Total $\mathrm{T}_{3}(\mathrm{ng} / \mathrm{dl})$ & $147.4(132.5-175.9)$ & $5-12$ \\
Total $\mathrm{T}_{4}(\mu \mathrm{g} / \mathrm{dl})$ & $10.2(8.8-11.7)$ & $12-23$ \\
TBG $(\mathrm{mg} / \mathrm{l})$ & $18.3 \pm 3.3$ & $>35 \mathrm{U} / \mathrm{l}:+$ auto- \\
ATPO $(\mathrm{U} / \mathrm{l})$ & $8.6(6.8-10.9)$ & $>115 \mathrm{U} / \mathrm{l}:+$ auto- \\
& & immunity \\
ATG $(\mathrm{U} / \mathrm{l})$ & $12.3(10-15.8)$ & \\
Percentage & $5 \%$ & \\
$\quad$ autoimmunity & & \\
\hline
\end{tabular}

Conversion factor for $\mathrm{FT}_{3}$ from $\mathrm{pg} / \mathrm{ml}$ to $\mathrm{pmol} / \mathrm{l}$ and for $\mathrm{TT}_{3}$ from $\mathrm{ng} / \mathrm{dl}$ to $\mathrm{nmol} / /$ is 0.0154 ; conversion factor for $\mathrm{FT}_{4}$ from $\mathrm{ng} / \mathrm{dl}$ to $\mathrm{pmol} / \mathrm{l}$ and for $\mathrm{TT}_{4}$ from $\mu \mathrm{g} / \mathrm{d}$ to $\mathrm{nmol} / \mathrm{l}$ is 12.87 .

The intra- and interassay CV \% were 1.0 and $6.1 \%$ for $\mathrm{FT}_{4}, 4.3$ and $2.9 \%$ for $\mathrm{FT}_{3}$, and 6.7 and $2.3 \%$ for $\mathrm{TSH}$ respectively. $\mathrm{TT}_{3}, \mathrm{TT}_{4}$, and $\mathrm{TBG}$ were measured using a RIA (DIAsource ImmunoAssays S.A., Nivelles, Belgium). The intra- and interassay CV were 4.7 and $3.7 \%$ for $\mathrm{TT}_{3}, 5.6$ and $6.5 \%$ for $\mathrm{TT}_{4}$, and 4 and $3.5 \%$ for TBG respectively.

\section{Statistics}

Descriptives are expressed as mean \pm s.D. or median (first to third quartile) when criteria for normality were not fulfilled (Kolmogorov-Smirnov) and variables (bone parameters and hormone concentrations) were $\log$ transformed in subsequent linear models. Linear mixed-effects modeling was used to evaluate crosssectional relationships in our study population, taking the interdependence of measurements within families into account. All analyses considering bone mass were adjusted for age, height, weight, and smoking status. Parameters of fixed effects were estimated via restricted maximum likelihood estimation and reported as standardized estimates of effect size $(\beta)$ with their respective standard error. Associations were considered significant at $P$ values $<0.05$. Statistical analyses were performed using Spotfire S+8.1 (Insightful, Seattle, WA, USA) and Medcalc 11 (Mariakerke, Belgium). The formula for $R^{2}$ of Cox and Snell was used to calculate the proportion of variation explained by $\mathrm{FT}_{3}$ and TBG.

\section{Results}

\section{General characteristics and thyroid hormone status}

The general characteristics, thyroid parameters, and pQCT measurements of the whole study population $(n=677)$ are shown in Tables 1 and 2. Based on the exclusion criteria, subjects with thyroid disease were excluded a priori. We additionally excluded subjects with positive thyroid autoimmunity after determination of thyroid antibodies (TPOAb $>35 \mathrm{U} / \mathrm{l}$ or $\mathrm{TgAb}>115 \mathrm{U} / \mathrm{l}$ ), leaving 641 subjects. No differences in anthropometrics were observed between subjects with and without thyroid autoimmunity.

$\mathrm{TT}_{4}$ was strongly $(\beta=0.51 \pm 0.03, P<0.0001)$ and $\mathrm{FT}_{4}$ was not associated with TBG $(\beta=-0.002$, $P=0.95) . \mathrm{TT}_{3}$ was also strongly associated with TBG $(\beta=0.56 \pm 0.03, P<0.0001)$, as well as $\mathrm{FT}_{3}(\beta=0.17$ $\pm 0.03, P<0.0001)$. Significant positive associations between free thyroid hormones and ratios of total hormones to TBG were observed $(\beta=0.24 \pm 0.04$, $P<0.0001)$. No differences in anthropometrics were observed between subjects with and without thyroid autoimmunity.

\section{Thyroid function in healthy young men in relation to age, body composition, and lifestyle factors}

No effects of age, body height, or weight were observed on TSH, whereas free thyroid hormone concentrations decreased with age $\left(\mathrm{FT}_{4}: \beta=-0.18 \pm 0.04, P<0.001\right.$; $\left.\mathrm{FT}_{3}: \beta=-0.14 \pm 0.04, P<0.001\right)$. TBG concentrations were positively associated with BMI $(\beta=0.13 \pm 0.04$, $P=0.001$ ) but not with age. $\mathrm{FT}_{4}$, but not $\mathrm{TT}_{4}$, concentrations were positively associated with body height ( $\beta=0.09 \pm 0.04, P=0.03)$, whereas no effect of body weight on $\mathrm{FT}_{4}$ or $\mathrm{TT}_{4}$ was observed. $\mathrm{FT}_{3}$ and $\mathrm{TT}_{3}$ were positively related to body weight $(\beta=0.09 \pm 0.04$, $P=0.03$ and $\beta=0.1 \pm 0.04, P=0.01$ respectively).

Current smokers displayed higher $\mathrm{FT}_{4}(1.49 \pm 0.20$ vs $1.43 \pm 0.18 \mathrm{ng} / \mathrm{dl}, P=0.002)$, higher $\mathrm{FT}_{3}(4.04$ \pm 0.37 vs $3.87 \pm 0.35 \mathrm{pg} / \mathrm{ml}, P<0.001)$, and lower TSH (1.4 (1.05-1.94) vs $1.53(1.18-2.06) \mu \mathrm{U} / \mathrm{l}$, $P=0.04)$ compared with nonsmokers or previous

Table 2 Descriptives of peripheral quantitative computed tomography bone parameters of study participants $(n=677)$ before exclusion of persons with positive autoimmunity.

\begin{tabular}{lc}
\hline Radius & \\
Cortical bone area $\left(\mathrm{mm}^{2}\right)$ & $101.3 \pm 13.6$ \\
Cortical bone density $\left(\mathrm{mg} / \mathrm{cm}^{3}\right)$ & $1100 \pm 35$ \\
Trabecular bone area $\left.(\mathrm{mm})^{2}\right)$ & $185.4 \pm 26.1$ \\
Trabecular bone density $\left(\mathrm{mg} / \mathrm{cm}^{3}\right)$ & $228 \pm 40$ \\
Tibia & $364 \pm 47$ \\
Cortical bone area $\left(\mathrm{mm}^{2}\right)$ & $1112 \pm 24$ \\
Cortical bone density $\left(\mathrm{mg} / \mathrm{cm}^{3}\right)$ & \\
\hline
\end{tabular}



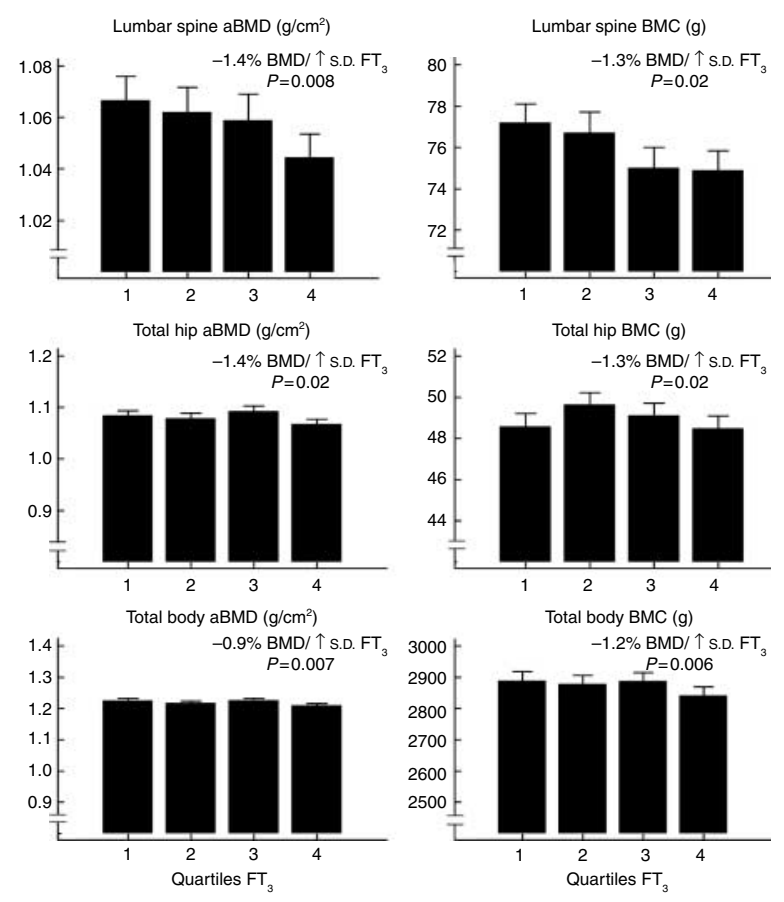

Figure $1 \mathrm{aBMD}$ and $\mathrm{BMC}$ according to quartiles of $\mathrm{FT}_{3}$.

smokers. All further analyses were adjusted for age, body height, weight, and smoking.

\section{Thyroid hormone status in relation to areal bone mass (DXA)}

We observed inverse associations of both $\mathrm{FT}_{3}$ and $\mathrm{TT}_{3}$ with bone mineral content (BMC) at the spine, the hip, as well as the whole body. In addition, areal BMD (aBMD) at both the level of the hip and the whole body was inversely associated with $\mathrm{FT}_{3}$ and $\mathrm{TT}_{3}$. At the level of the spine, aBMD was also inversely related to $\mathrm{FT}_{3}$, but a similar trend for $\mathrm{TT}_{3}$ was only borderline significant (Fig. 1, Table 3). $\mathrm{FT}_{3}$ explained $0.52 \%$ of variation of hip BMD and $0.41 \%$ for BMD at the spine, when adjusted for age, length, weight, and smoking status.
Similarly, $\mathrm{TT}_{4}$ was inversely associated with $\mathrm{BMC}$ at the three measurement sites, and with aBMD at the level of the hip and the total body (Table 3).

There was no significant relationship between either $\mathrm{FT}_{4}$ or TSH and BMC or BMD as measured by DXA (Table 3).

We observed a strongly negative association between TBG and DXA parameters at all levels (Table 3). When adjusted for age, length, weight, and smoking status, TBG explained $2.6 \%$ of variation in total hip BMD, $0.66 \%$ of variation in BMD at the spine, and $1.6 \%$ of variation for the whole body. When introducing TBG in our statistical model as a covariate, only $\mathrm{FT}_{3}$ remained significantly associated with aBMD at spine, hip, and whole body, although this association was weakened. The associations between total thyroid hormones and DXA measurements after adjustment for TBG were no longer significant (Data not shown).

\section{Thyroid hormones in relation to bone geometry and volumetric bone density}

The associations between thyroid hormones and pQCT-derived bone parameters are shown in Fig. 2. At the radius, cortical bone area was inversely associated with $\mathrm{TT}_{3}(\beta=-0.09 \pm 0.04, P=0.01), \mathrm{TT}_{4}(\beta=-0.09$ $\pm 0.04, \quad P=0.01)$, and TBG $(\beta=-0.12 \pm 0.04$, $P=0.0008)$ and trabecular bone density was inversely associated with $\mathrm{FT}_{4}(\beta=-0.08 \pm 0.04, P=0.04), \mathrm{TT}_{4}$ $(\beta=-0.1 \pm 0.04, P=0.005)$, and TBG $(\beta=-0.13$ $\pm 0.04, P=0.001)$. Inverse associations between cortical bone area at the mid-tibia and $\mathrm{FT}_{3}(\beta=-0.08$ $\pm 0.04, P=0.02), \mathrm{TT}_{3}(\beta=-0.12 \pm 0.04, P=0.0006)$, $\mathrm{TT}_{4}(\beta=-0.10 \pm 0.03, P=0.003)$, and TBG $(\beta=$ $-0.14 \pm 0.04, P=0.0001)$ were observed. However, the stronger described associations between total thyroid hormones and cortical bone area and trabecular bone density disappeared when TBG was added to the model as a covariate, similar to our observations with DXA measurements (data not shown).

Table 3 Relationship between TSH, thyroid hormone concentrations, thyroid binding globulin, and dual-energy x-ray absorptiometry bone parameters. Results from mixed effects models to account for family structure and adjusted for age, height, weight, and smoking, after exclusion of positive thyroid auto immunity. Data are presented as standardized estimates \pm S.E.M.

\begin{tabular}{lllllll}
\hline & \multicolumn{1}{c}{ TSH $(\mu \mathrm{U} / \mathrm{ml})$} & $\mathbf{F T}_{\mathbf{3}}(\mathrm{pg} / \mathrm{ml})$ & $\mathbf{T T}_{\mathbf{3}}(\mathrm{ng} / \mathrm{dl})$ & $\mathbf{F T}_{\mathbf{4}}(\mathrm{ng} / \mathrm{dl})$ & $\mathrm{TT}_{\mathbf{4}}(\mu \mathrm{g} / \mathrm{dl})$ & TBG $(\mathrm{mg} / \mathrm{dl})$ \\
\hline aBMD lumbar spine & $\beta=-0.003 \pm 0.04$ & $\beta=-0.10 \pm 0.04$ & $\beta=-0.08 \pm 0.04$ & $\beta=-0.03 \pm 0.04$ & $\beta=-0.05 \pm 0.04$ & $\beta=-0.12 \pm 0.04$ \\
$\left(\mathrm{~g} / \mathrm{cm}^{2}\right)$ & $P=0.94$ & $P=0.008$ & $P=0.05$ & $P=0.5$ & $P=0.2$ & $P=0.003$ \\
aBMD total hip & $\beta=0.03 \pm 0.03$ & $\beta=-0.08 \pm 0.03$ & $\beta=-0.14 \pm 0.04$ & $\beta=-0.05 \pm 0.04$ & $\beta=-0.11 \pm 0.04$ & $\beta=-0.17 \pm 0.04$ \\
$\left(\mathrm{~g} / \mathrm{cm}^{2}\right)$ & $P=0.40$ & $P=0.02$ & $P=0.002$ & $P=0.15$ & $P=0.002$ & $P<0.0001$ \\
aBMD whole body & $\beta=0.008 \pm 0.04$ & $\beta=-0.09 \pm 0.03$ & $\beta=-0.11 \pm 0.04$ & $\beta=-0.07 \pm 0.04$ & $\beta=-0.1 \pm 0.04$ & $\beta=-0.14 \pm 0.04$ \\
$\left(\mathrm{~g} / \mathrm{cm}^{2}\right)$ & $P=0.83$ & $P=0.007$ & $P=0.01$ & $P=0.053$ & $P=0.006$ & $P=0.0001$ \\
BMC lumbar spine (g) & $\beta=-0.005 \pm 0.03$ & $\beta=-0.08 \pm 0.03$ & $\beta=-0.12 \pm 0.05$ & $\beta=-0.03 \pm 0.04$ & $\beta=-0.07 \pm 0.04$ & $\beta=-0.15 \pm 0.04$ \\
& $P=0.90$ & $P=0.02$ & $P=0.009$ & $P=0.5$ & $P=0.004$ & $P<0.0001$ \\
BMC total hip (g) & $\beta=0.04 \pm 0.03$ & $\beta=-0.08 \pm 0.03$ & $\beta=-0.15 \pm 0.05$ & $\beta=-0.05 \pm 0.04$ & $\beta=-0.1 \pm 0.03$ & $\beta=-0.16 \pm 0.03$ \\
& $P=0.29$ & $P=0.02$ & $P=0.002$ & $P=0.16$ & $P=0.002$ & $P<0.0001$ \\
BMC whole body (g) & $\beta=0.01 \pm 0.03$ & $\beta=-0.08 \pm 0.03$ & $\beta=-0.14 \pm 0.05$ & $\beta=-0.04 \pm 0.03$ & $\beta=-0.08 \pm 0.03$ & $\beta=-0.14 \pm 0.03$ \\
& $P=0.64$ & $P=0.006$ & $P=0.004$ & $P=0.20$ & $P=0.01$ & $P<0.0001$ \\
\hline
\end{tabular}




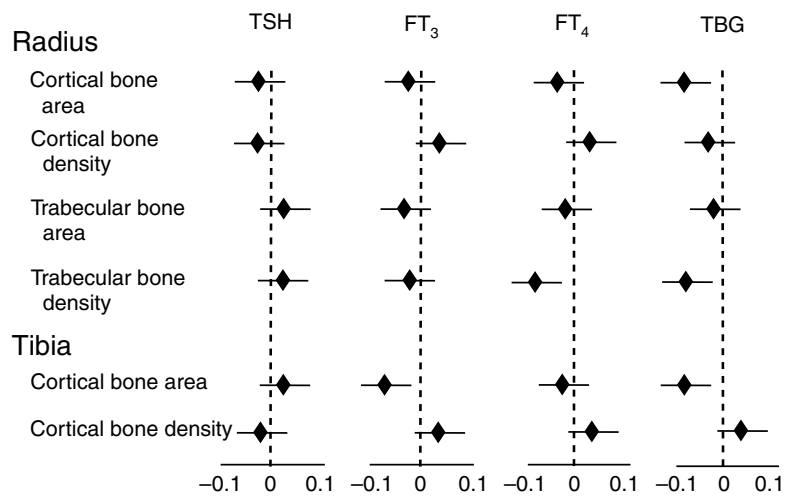

Figure 2 Standardized estimates representing associations between $\mathrm{TSH}, \mathrm{FT}_{3}, \mathrm{FT}_{4}$, and TBG and cortical and trabecular bone area and density, adjusted for age, height, weight, and current smoking at the radius and tibia (bar $=95 \%$ confidence limit).

No associations between TSH and pQCT-derived bone parameters were observed (Fig. 2).

\section{Thyroid hormones in relation to bone turnover markers}

There was no relationship between biochemical markers of bone formation (P1NP) or resorption (CTX) and free or total thyroid hormones and TSH (e.g. $\mathrm{FT}_{3}$ and P1NP: $P=0.6$ ). The higher described association between $\mathrm{FT}_{3}$ and $\mathrm{aBMD}$ and $\mathrm{BMC}$ did not change when adding markers of bone turnover as a covariate (e.g. addition of P1NP: aBMD hip and $\mathrm{FT}_{3}: P=0.005$, aBMD spine and $\mathrm{FT}_{3}: P=0.004$, aBMD total body and $\left.\mathrm{FT}_{3}: P=0.007\right)$.

\section{TBG in relation to sex steroid hormones and SHBG}

In order to explore possible mechanisms underlying the association between TBG and bone parameters, we investigated the relationship between sex steroids and TBG. No associations between estrogens (free and total) or testosterone (free and total) and TBG were observed. The associations between TBG and DXA and pQCT measurements remained when sex steroids were added to the model (data not shown). The negative associations between TBG and DXA and pQCT parameters remained intact when SHBG was added to the model as a covariate.

\section{Discussion}

This study demonstrates that between-subject variation in thyroid hormone status within the physiological range is related to $\mathrm{aBMD}$ and $\mathrm{VBMD}$ in healthy men at the age of PBM. Higher $\mathrm{FT}_{3}, \mathrm{TT}_{3}, \mathrm{TT}_{4}$, and $\mathrm{TBG}$ are associated with lower aBMD and BMC at various skeletal sites. However, after correction for TBG, only $\mathrm{FT}_{3}$ remains significantly associated with aBMD. Measuring vBMD and bone geometry by pQCT, we observe inverse relationships between $\mathrm{FT}_{4}, \mathrm{TT}_{4}$, and TBG and trabecular bone density as well as inverse associations between $\mathrm{FT}_{3}, \mathrm{TT}_{3}, \mathrm{TT}_{4}$, and $\mathrm{TBG}$ and cortical bone area.

The hormonal determinants of bone mass in men have not been fully investigated. Whereas our current understanding on the effects of thyroid hormone status on the skeleton in the adult is merely based on the studies in postmenopausal women or in situations characterized by thyroid dysfunction, this is the first study that considers young men at the age of PBM. Moreover, this report describes relationships between thyroid status and bone parameters determined not only by two-dimensional areal DXA estimations but also by three-dimensional geometric and volumetric pQCT measurements in men.

Since von Recklinghausen first described a case of thyrotoxic bone disease in 1891, hyperthyroidism is a well-known cause of osteopenia and osteoporosis (18). Studies on the relation between hyperthyroidism, aBMD, and fracture risk confirm a decrease of aBMD and an increase of fracture risk. This risk augments with advancing age and returns to normal upon treatment of the hyperthyroid state $(9,19,20)$. Untreated subclinical hyperthyroidism in postmenopausal women has also been associated with decreased aBMD and a higher fracture risk compared with women who received treatment, although findings have not been unequivocal (21-24). Little data exists on the influence of subclinical hyperthyroidism on the skeleton in men (5).

Thyrotoxicosis results in increased global bone turnover as indicated by elevated values of the biochemical markers of bone formation and bone resorption (5), which in general correlate well with thyroid hormone concentrations, especially in cases of (subclinical) hyperthyroidism (3). Furthermore, histomorphometric data indicate that excessive thyroid hormone results in a shortening of the different phases of the remodeling cycle and an $\sim 10 \%$ relative deficit of bone formation per cycle (8). Therefore, the decrease in BMD observed in thyrotoxicosis is explained both by an (reversible) expansion of the so-called remodeling space, with increased cortical porosity, and by accelerated bone loss that might primarily affect trabecular bone. Conversely, hypothyroidism is characterized by a lower global bone turnover and prolongation of the remodeling cycle with a small positive bone balance per cycle. When situations of excessive or deficient thyroid hormone are prolonged, the effects on bone remodeling can potentially result in a decreased or increased mineralization degree of bone tissue respectively $(7,8)$.

This study differentiates from most clinical data on the relationship between thyroid and skeletal status first in that we considered young men at the age of PBM and secondly in that we studied the influence of variations in 
thyroid hormone status within the physiological range. Therefore, we excluded subjects with a history of thyroid disease or treatment with thyroid hormone or with positive levels of thyroid autoantibodies from our analyses. We observed an inverse relationship between $\mathrm{FT}_{4}$ and $\log \mathrm{TSH}$ in our cohort, but this association was no longer significant after exclusion of subjects with thyroid autoimmunity, compatible with the premise that we studied euthyroid subjects. Our study differs from the preceding studies because we also investigated associations between thyroid hormone levels and volumetric bone density and cross-sectional bone geometry. Our data show that thyroid status is associated not only with trabecular bone density but also with bone size (cortical bone area).

The novelty of our results is that between-subject physiological variation in apparent thyroid hormone exposure is associated with skeletal characteristics in men at the age of PBM and that higher levels of thyroid hormones, even within the normal range, have a negative influence on parameters of bone strength at this young adult age. The negative association between thyroid hormone levels and aBMD is seen for all assessed skeletal sites, although most consistently for $\mathrm{FT}_{3}$.

In line with our findings in young men, van der Deure et al. (14) reported in elderly men and women a negative association between $\mathrm{FT}_{4}$ and aBMD and, when corrected for BMI, no significant association with TSH, although there was a trend (for a positive relationship between TSH and aBMD). However, $\mathrm{FT}_{3}$ was not determined in their study. Murphy et al. (13) found that both higher $\mathrm{FT}_{4}$ and $\mathrm{FT}_{3}$ were associated with reduced aBMD in euthyroid postmenopausal women. We found associations only between $\mathrm{FT}_{3}$ and BMD or BMC, but not with $\mathrm{FT}_{4}$, which is in agreement with the premise that most actions of thyroid hormone in the body are mediated by the active form of thyroid hormone, $\mathrm{T}_{3}$. More specifically in bone, remodeling is considered to be predominantly mediated by via $\mathrm{TR} \alpha(25)$.

However, expression of deiodinases in osteoblasts has been demonstrated (26). Not only circulating serum $\mathrm{T}_{3}$ but also local production of $\mathrm{T}_{3}$ in bone can exert effects on bone. Nevertheless, other regulatory mechanisms can play a role and the local production of $\mathrm{T}_{3}$ is dependent on varying concentrations of serum $\mathrm{T}_{4}$. Our data provides evidence for a negative effect of circulating $\mathrm{T}_{3}$ on bone, though this does not exclude local effects of deiodinase activity in bone, apart from the described systemic effects.

Because of the observation of significant negative associations between both total thyroid hormones and TBG and bone parameters, the question raised was if TBG could be the main mediator for the associations observed with total thyroid hormones. Indeed, after correction for TBG, the associations between total thyroid hormones and DXA and pQCT parameters became insignificant. We hypothesized that the effects of TBG on bone might be mediated by sex steroids, because administration of estrogens and androgens is known to be associated with an increase and decrease in the level of TBG respectively (27). However, in our study, TBG was not associated with the levels of free or total endogenous estrogen or testosterone. Furthermore, the stronger described associations between TBG and bone parameters remained significant when sex steroids or SHBG were introduced to our model as covariates. Moreover, the associations of TBG and bone parameters were independent of SHBG and appeared to be associated more strongly to bone than SHBG. Another argument in favor of an independent role for TBG apart from sex steroids is the consistently negative association between TBG and bone parameters, whereas associations between sex steroids $\left(\mathrm{E}_{2}\right)$ or SHBG and bone parameters are merely positive.

As TBG is negatively correlated with thyroid state (it decreases in hyperthyroidism and increases in hypothyroidism) $(28,29)$, one would theoretically expect that the observed relationship with bone would be different. Nevertheless, the associations between TBG and bone parameters are in the same direction as the thyroid hormones themselves (negatively), as is the case with SHBG and sex steroids (positively). In this regard, the binding proteins seem to potentiate the effect of their corresponding free hormones. Underlying these observations, a hypothetical transporter, facilitating hormone transfer across cellular membranes, could be active. However, no transporter has been described for TBG and the endocytic megalin-carrier pathway for SHBG remains unproven. TBG could also be a marker for nutritional status because we indeed observed a positive relation between TBG and BMI, but a positive relationship between TBG and bone parameters would be expected and malnutrition was not present in our population of healthy young men.

Further studies should be designed to elucidate whether TBG is a real determinant of bone mass or only a marker of another unknown determinant affecting bone.

This association study does not allow to establish a causal relationship or to unravel underlying pathophysiological mechanisms. In the context of our primary focus of interest, i.e. the determinants of PBM in men, one of the major questions raised by our findings is whether the recorded associations reflect actual effects of thyroid hormone on bone homeostasis in these adult young men and/or earlier effects of thyroid hormone that occurred during bone accrual. An argument in favor of the latter possibility might be provided by the fact that we could not observe an association between thyroid hormone levels and biochemical markers of bone turnover and no effect of these markers on the relation between thyroid hormones and aBMD. However, values for biochemical markers of bone turnover are rather high, with a broad range in men in their twenties before decreasing to a nadir toward the 
age of fifties $(30,31)$. It is thus possible that an effect of thyroid hormone on these variables has been obscured by other stronger influences. Another argument in favor of effects of thyroid hormone action on bone acquisition and maturation is our observation of associations of thyroid hormone levels with pQCTderived geometric bone parameters. Changes of the periosteal circumference by periosteal modeling occur essentially and rapidly during growth but are slow processes in young adulthood (32).

Recently, some authors have challenged the conventional view that skeletal responses to abnormal thyroid status result solely from altered $\mathrm{T}_{3}$ action in the bone and have proposed TSH as a negative regulator of bone turnover (23, 33-37). For example, Morris et al. (35) and Kim et al. (23) observed associations between low-normal serum TSH levels and osteopenia and osteoporosis prevalence, together with a graded increase in aBMD with increasing TSH in postmenopausal women. van der Deure et al. (14) found in the Rotterdam study - a population of elderly Caucasians that femoral neck aBMD as well as a DXA derived cortical thickness estimation increased with serum TSH, but this relationship became non-significant when corrected for BMI. Mazzioti et al. (37) observed that TSH values in the lower part of the normal range were associated with vertebral fractures in euthyroid postmenopausal women, independently of $\mathrm{FT}_{4}$, aBMD, and age. These findings are opposed by results from the Tromsø and the HUNT 2 study, where, as in our population, no relation of TSH with aBMD was observed within the normal range of serum $\operatorname{TSH}(34,36)$.

Moreover, these epidemiological studies cannot differentiate between the effects of excess thyroid hormone or TSH deficiency because the hypothalamopituitary-thyroid axis with its feedback regulation remains intact (38). Despite the demonstration of TSH receptor expression in osteoblasts and osteoclasts (33), in vitro studies have not established a clear and consistent role for TSH in bone cells so far. In contrast, elegant studies in mice provide strong arguments against direct effects of TSH on bone. Bassett et al. (38) observed that deletion of TR $\alpha$ in mice resulted in decreased $\mathrm{T}_{3}$ action in bone without affecting systemic thyroid status. Delayed ossification and osteosclerosis indicate that responses to reduced $\mathrm{T}_{3}$ action in bone predominate when TSH levels are normal. Similarly, mutation or deletion of TR $\beta$ results in increased $T_{3}$ action in bone and increased levels of TSH. Accelerated ossification and osteoporosis confirms that the effects of $\mathrm{T}_{3}$ excess predominate even when TSH levels are also increased, providing further evidence supporting that skeletal responses to hypo- and hyperthyroidism are mediated by $\mathrm{T}_{3}$ acting via $\mathrm{TR} \alpha$ and not by a direct effect of TSH on bone. In our cohort of young men, we could not find any relation between TSH and aBMD, BMC or volumetric trabecular and cortical density nor bone geometry, hereby supporting the conventional view.
We can conclude from this study that between-subject variation of thyroid hormones in the physiological range has an effect on bone mass, density, and geometry in healthy young men at the age of PBM, with higher levels of $\mathrm{FT}_{3}, \mathrm{TT}_{3}, \mathrm{TT}_{4}$, and $\mathrm{TBG}$ being associated with lower $\mathrm{aBMD}$ and $\mathrm{BMC}$ at various skeletal sites.

\section{Declaration of interest}

The authors declare that there is no conflict of interest that could be perceived as prejudicing the impartiality of the research reported.

\section{Funding}

This study is supported by a grant from the Fund for Scientific Research - Flanders (FWO-Vlaanderen grant \#G.0662.07). Y Taes holds a postdoctoral fellowship of the Research Foundation - Flanders (FWO). Unrestricted research grant from Servier Benelux.

\section{Acknowledgements}

The authors are indebted to K Toye, K Mertens, E Vandersypt, and M Becqué for their excellent technical assistance.

\section{References}

1 Valimaki MJ, Karkkainen M, Lamberg-Allardt C, Laitinen K, Alhava E, Heikkinen J, Impivaara O, Makela P, Palmgren J \& Seppanen R. Exercise, smoking, and calcium intake during adolescence and early adulthood as determinants of peak bone mass. Cardiovascular Risk in Young Finns Study Group. BMJ 1994 $309230-235$.

2 Taes YE, Lapauw B, Vanbillemont G, Bogaert V, De Bacquer D, Zmierczak H, Goemaere S \& Kaufman JM. Fat mass is negatively associated with cortical bone size in young healthy male siblings. Journal of Clinical Endocrinology and Metabolism $2009 \mathbf{9 4}$ 2325-2331. (doi:10.1210/jc.2008-2501)

3 Lapauw BM, Taes Y, Bogaert V, Vanbillemont G, Goemaere S, Zmierczak HG, De Bacquer D \& Kaufman JM. Serum estradiol is associated with volumetric BMD and modulates the impact of physical activity on bone size at the age of peak bone mass: a study in healthy male siblings. Journal of Bone and Mineral Research 2009 24 1075-1085. (doi:10.1359/jbmr.081260)

4 Lapauw B, Taes Y, Goemaere S, Toye K, Zmierczak HG \& Kaufman JM. Anthropometric and skeletal phenotype in men with idiopathic osteoporosis and their sons is consistent with deficient estrogen action during maturation. Journal of Clinical Endocrinology and Metabolism 200994 4300-4308. (doi:10. 1210/jc.2009-0568)

5 Murphy E \& Williams GR. The thyroid and the skeleton. Clinical Endocrinology 200461 285-298. (doi:10.1111/j.1365-2265. 2004.02053.x)

6 Williams GR. Actions of thyroid hormones in bone. Endokrynologia Polska 200960 380-388.

7 Eriksen EF. Normal and pathological remodeling of human trabecular bone: three dimensional reconstruction of the remodeling sequence in normals and in metabolic bone disease. Endocrine Reviews 19867 379-408. (doi:10.1210/edrv-7-4-379)

8 Mosekilde L, Eriksen EF \& Charles P. Effects of thyroid hormones on bone and mineral metabolism. Endocrinology and Metabolism Clinics of North America 199019 35-63.

9 Vestergaard P \& Mosekilde L. Hyperthyroidism, bone mineral, and fracture risk - a meta-analysis. Thyroid 200313 585-593. (doi:10.1089/105072503322238854) 
10 Lakatos P. Thyroid hormones: beneficial or deleterious for bone? Calcified Tissue International 200373 205-209. (doi:10.1007/ s00223-002-0027-8)

11 Karga H, Papapetrou PD, Korakovouni A, Papandroulaki F, Polymeris A \& Pampouras G. Bone mineral density in hyperthyroidism. Clinical Endocrinology 200461 466-472. (doi:10.1111/j. 1365-2265.2004.02110.x)

12 Vestergaard $\mathrm{P} \&$ Mosekilde L. Fractures in patients with hyperthyroidism and hypothyroidism: a nationwide follow-up study in 16249 patients. Thyroid 200212 411-419. (doi:10. 1089/105072502760043503)

13 Murphy E, Gluer CC, Reid DM, Felsenberg D, Roux C, Eastell R \& Williams GR. Thyroid function within the upper normal range is associated with reduced bone mineral density and an increased risk of nonvertebral fractures in healthy euthyroid postmenopausal women. Journal of Clinical Endocrinology and Metabolism 2010 95 3173-3181. (doi:10.1210/jc.2009-2630)

14 van der Deure WM, Uitterlinden AG, Hofman A, Rivadeneira F, Pols HA, Peeters RP \& Visser TJ. Effects of serum TSH and $\mathrm{FT}_{4}$ levels and the TSHR-Asp727Glu polymorphism on bone: the Rotterdam Study. Clinical Endocrinology 200868 175-181. (doi:10.1111/j.1365-2265.2007.03016.x)

15 Taes Y, Lapauw B, Vanbillemont G, Bogaert V, De Bacquer D, Goemaere S, Zmierczak H \& Kaufman JM. Early smoking is associated with peak bone mass and prevalent fractures in young healthy men. Journal of Bone and Mineral Research 200925 379387. (doi:10.1359/jbmr.090809)

16 Lohman T, Roche A, Martorell R. Anthropometric Standardization Reference Manual, 1 edn. Champaign, IL, USA: Human Kinetics Books, 1988.

17 Thienpont LM, Van Uytfanghe K, Beastall G, Faix JD, Ieiri T, Miller WG, Nelson JC, Ronin C, Ross HA, Thijssen JH \& Toussaint B. Report of the IFCC Working Group for Standardization of Thyroid Function Tests; part 2: free thyroxine and free triiodothyronine. Clinical Chemistry 2010 56 912-920. (doi:10. 1373/clinchem.2009.140194)

18 Von Recklinghausen FD. Die Fibröse oder deformierende Ostitis, die Osteomalazie und die osteoplastische Carzinose in ihren gegenseitigen Beziehungen. Berlin, 1891.

19 Rosen CJ \& Adler RA. Longitudinal changes in lumbar bone density among thyrotoxic patients after attainment of euthyroidism. Journal of Clinical Endocrinology and Metabolism $1992 \mathbf{7 5}$ 1531-1534. (doi:10.1210/jc.75.6.1531)

20 Grant DJ, McMurdo ME, Mole PA \& Paterson CR. Is previous hyperthyroidism still a risk factor for osteoporosis in postmenopausal women? Clinical Endocrinology 199543 339-345. (doi:10.1111/j.1365-2265.1995.tb02041.x)

21 Bauer DC, Nevitt MC, Ettinger B \& Stone K. Low thyrotropin levels are not associated with bone loss in older women: a prospective study. Journal of Clinical Endocrinology and Metabolism $1997 \mathbf{8 2}$ 2931-2936. (doi:10.1210/jc.82.9.2931)

22 Bauer DC, Ettinger B, Nevitt MC \& Stone KL. Risk for fracture in women with low serum levels of thyroid-stimulating hormone. Annals of Internal Medicine 2001134 561-568.

23 Kim DJ, Khang YH, Koh JM, Shong YK \& Kim GS. Low normal TSH levels are associated with low bone mineral density in healthy postmenopausal women. Clinical Endocrinology 2006 64 86-90. (doi:10.1111/j.1365-2265.2005.02422.x)

24 Ross DS. Hyperthyroidism, thyroid hormone therapy, and bone. Thyroid 1994 4 319-326. (doi:10.1089/thy.1994.4.319)
25 Heemstra KA, Hoftijzer $H$, van der Deure WM, Peeters RP, Hamdy NA, Pereira A, Corssmit EP, Romijn JA, Visser TJ \& Smit JW. The type 2 deiodinase Thr92Ala polymorphism is associated with increased bone turn-over and decreased femoral neck bone mineral density. Journal of Bone and Mineral Research 201025 1385-1391. (doi:10.1002/jbmr.27)

26 Williams AJ, Robson H, Kester MH, van Leeuwen JP, Shalet SM, Visser TJ \& Williams GR. Iodothyronine deiodinase enzyme activities in bone. Bone 200843 126-134. (doi:10.1016/j.bone. 2008.03.019)

27 Tahboub R \& Arafah BM. Sex steroids and the thyroid. Best Practice and Research. Clinical Endocrinology and Metabolism 2009 23 769-780. (doi:10.1016/j.beem.2009.06.005)

28 Rudorff KH. Thyroxine-binding globulin (TBG). Clinical studies on the regulation of TBG concentration in serum and the value of TBG for the evaluation of thyroid function. Fortschritte der Medizin $1979972038-2045$.

29 Inada M \& Sterling K. Thyroxine transport in thyrotoxicosis and hypothyroidism. Journal of Clinical Investigations $1967 \mathbf{4 6}$ 1442-1450. (doi:10.1172/JCI105636)

30 Seibel MJ. Biochemical markers of bone turnover: part I: biochemistry and variability. Journal of Clinical Investigation 2005 26 97-122.

31 Szulc P, Kaufman JM \& Delmas PD. Biochemical assessment of bone turnover and bone fragility in men. Osteoporosis International 200718 1451-1461. (doi:10.1007/s00198-0070407-z)

32 Szulc P \& Seeman E. Thinking inside and outside the envelopes of bone: dedicated to PDD. Osteoporosis International 200920 1281-1288. (doi:10.1007/s00198-009-0994-y)

33 Abe E, Marians RC, Yu W, Wu XB, Ando T, Li Y, Iqbal J, Eldeiry L, Rajendren G, Blair HC, Davies TF \& Zaidi M. TSH is a negative regulator of skeletal remodeling. Cell $2003 \mathbf{1 1 5}$ 151-162. (doi:10.1016/S0092-8674(03)00771-2)

34 Grimnes G, Emaus N, Joakimsen RM, Figenschau Y \& Jorde R. The relationship between serum TSH and bone mineral density in men and postmenopausal women: the Tromso study. Thyroid $2008 \mathbf{1 8}$ 1147-1155. (doi:10.1089/thy.2008.0158)

35 Morris MS. The association between serum thyroid-stimulating hormone in its reference range and bone status in postmenopausal American women. Bone 200740 1128-1134. (doi:10.1016/j. bone.2006.12.001)

36 Svare A, Nilsen TI, Bjoro T, Forsmo S, Schei B \& Langhammer A. Hyperthyroid levels of TSH correlate with low bone mineral density: the HUNT 2 study. European Journal of Endocrinology 2009 161 779-786. (doi:10.1530/EJE-09-0139)

37 Mazziotti G, Porcelli T, Patelli I, Vescovi PP \& Giustina A. Serum TSH values and risk of vertebral fractures in euthyroid postmenopausal women with low bone mineral density. Bone 201046 747-751. (doi:10.1016/j.bone.2009.10.031)

38 Bassett JH \& Williams GR. Critical role of the hypothalamicpituitary-thyroid axis in bone. Bone 200843 418-426. (doi:10. 1016/j.bone.2008.05.007)

Received 14 February 2011

Accepted 10 March 2011 